\title{
Organotypic Modeling of Human Keratinocyte Response to Peroxisome Proliferators
}

\author{
Carmen Zhang $^{\mathrm{a}}$ Igor Gurevich ${ }^{\mathrm{b}}$ Brian J. Aneskievich ${ }^{\mathrm{a}, \mathrm{c}}$ \\ ${ }^{a}$ Department of Pharmaceutical Sciences, and ${ }^{b}$ Graduate Program in Toxicology, School of Pharmacy, and \\ 'Stem Cell Institute, University of Connecticut, Storrs, Conn., USA
}

\section{Key Words}

Keratinocyte - Differentiation - Organotypic modeling •

Peroxisome proliferator $\cdot$ Peroxisome proliferator-activated receptor

\begin{abstract}
Peroxisome proliferators (PPs) are a diverse chemical group including hypolipidemic drugs and some fatty acids. Their stimulation of PP-activated receptors (PPARs) and subsequent control of gene expression regulates metabolism and differentiation in many cells. PPs have multiple opportunities to target human epidermal keratinocytes because of delivery through dietary, clinical, and/or topical exposure routes. PPAR knockout mice and PP treatment of mouse skin or human keratinocytes in monolayer culture have established some effects for PPs in cutaneous differentiation. However, incomplete epidermal maturation characteristic of monolayer keratinocytes and rodent-specific effects may limit our full understanding of human keratinocyte responses to PPs. To address these issues, we investigated PP influence on primary human keratinocytes in organotypic cultures that recapitulate biochemical markers of epidermis. We found that the PPAR $\alpha$ agonists clofibrate, docasohexaenoic acid, and WY-14,643 produced mild to moderate keratinocyte hyperplasia, increased stratification (particularly of
\end{abstract}

granular and cornified layers), and enhanced levels of the differentiation markers filaggrin, $A B C A 12$, and phosphorylated HSP27. Several PP effects generated in the organotypic system, however, were distinct from those previously reported for rodent skin and human keratinocyte monolayer cultures, suggesting that the species and growth context of target cells can impact exposure outcomes. Given the utility of organotypic cultures for modeling the epidermis, studies in this system may bridge the gap between the rodent assays and clinical studies of human epidermal responses to PPs.

Copyright $\odot 2012$ S. Karger AG, Basel

\section{Abbreviations used in this paper}

ABCA12 ATP-binding cassette, sub-family A (ABC1),

$\mathrm{ACO}$

CLF

DHA

PCNA

PP

PPAR

RA

RAR

RXR

WY member 12

acyl-CoA oxidase

clofibrate

docosahexaenoic acid

proliferating cell nuclear antigen

peroxisome proliferator

peroxisome proliferator-activated receptor

retinoic acid

retinoic acid receptor

retinoid $\mathrm{X}$ receptor

WY 14,643

\section{KARGER}

Fax +4161306 1234

E-Mail karger@karger.ch

www.karger.com
(C) 2012 S. Karger AG, Basel

$1422-6405 / 12 / 1965-0431 \$ 38.00 / 0$

Accessible online at:

www.karger.com/cto
Dr. Brian J. Aneskievich

University of Connecticut

69 North Eagleville Road, U-3092

Storrs, CT 06269 (USA)

Tel. +1 860486 3053, E-Mail brian.aneskievich@uconn.edu 


\section{Introduction}

Peroxisome proliferators (PPs) are a heterogeneous chemical group including agricultural and industrial compounds, hypolipidemic and insulin sensitizing drugs, and long chain polyunsaturated fatty acids that exert transcriptional effects through PP-activated receptors (PPARs) $\alpha, \delta$, and $\gamma$. Respectively, these groups include compounds such as certain pesticides and phthalate esters, clofibrate (CLF) and glitazones, and $\alpha$-linolenic and docosahexaenoic acids (DHA) [Takeuchi et al., 2006; Varga et al., 2011]. The widespread occurrence of PPs makes them of interest in cutaneous biology [see Schmuth et al., 2008, for review] because of their ample opportunity for epidermal contact through topical and systemic routes. Additionally, given their ability to control metabolism and differentiation, PPs in current medical use may be repurposed for use in wound healing following sufficient preclinical study. Despite this promise, certain caveats are raised by the studies done to date. For instance, fibrate compounds have extensive clinical use to lower human serum triglycerides [Michalik et al., 2006] despite classic and mechanistic causation of rodent liver cancer [Peters et al., 2005; Gonzalez and Shah, 2008] among other rodent species-specific results not seen in human cells [Choudhury et al., 2000; Klaunig et al., 2003]. Species differences extend to PPAR $\alpha$ amino acid differences, possibly affecting ligand binding. Also, PPAR $\alpha$ expression levels are higher in rodent liver compared to human liver [Keller et al., 1997; Maloney and Waxman, 1999]. The latter does not wholly resolve the issue; increasing PPAR $\alpha$ expression in human liver cells does not confer rodent cell-like responsiveness [Lawrence et al., 2001, and references therein]. The need to determine species-matched responses led to genetically modified mouse strains [Cheung et al., 2004; Morimura et al., 2006] 'humanized' for PPAR $\alpha$ such as those mice expressing human PPAR $\alpha$ in a PPAR $\alpha$ knockout background. However, this approach does not negate the variation across species sometimes found in the ligand metabolism or promoter sequence of PPAR target genes [Kane et al., 2006]. For epidermal studies, PPAR distribution also needs to be considered. In human skin, all three PPAR subtypes have been detected in keratinocytes, making its distribution distinct from adult mouse interfollicular epidermis where no PPAR expression is detectable [Westergaard et al., 2001; Michalik and Wahli, 2007]. Despite advantages of working with in vivo mouse skin or the selective genetic backgrounds of knockout or transgenic mice, rodent systems have limitations that might be avoided with the use of optimized human keratinocyte systems such as in vitro generated skin models.

Organotypic human keratinocyte cultures recapitulate the structural and biochemical markers of late epidermal differentiation [Margulis et al., 2005] that are usually missing from standard keratinocyte cultures grown on plastic substrates. Organotypic systems are a co-culture of keratinocytes at the air-liquid interface overlying an artificial dermis composed of fibroblasts and gelled collagen with nutrient support from media under the collagen layer. Although Chong et al. [2009] recently reported a PPAR $\delta$-mediated epithelial-mesenchymal interaction based on the receptor's regulation of cytokine expression in fibroblasts of organotypic cultures, direct responses of human keratinocytes to PPs in such models have not been thoroughly investigated. Previously, Rivier et al. [2000] showed that, similar to intact human skin, PPAR $\alpha$ is detected in human keratinocyte nuclei in organotypic cultures; these cultures showed enhanced lipid deposition along with increased expression of enzymes associated with lipid metabolism when treated with WY 14,643 (WY). Similar results were recently found with CLF [Batheja et al., 2009] although changes in structural proteins and other differentiation markers were not investigated in either study. Here, our aim was to use the organotypic culture system to examine human keratinocyte differentiation and growth responses to PPs and to analyze the results in the context of what has been previously reported for intact rodent skin and submerged keratinocyte cultures. We found an unexpected increase in keratinocyte replication along with some late stage differentiation enhancement, results which would not have been predicted based on rodent skin or submerged keratinocyte studies. Further study of the organotypic system could bridge the preclinical analysis gap for response to PPs between the incomplete differentiation of submerged keratinocytes on plastic and possible species-specific responses in rodent skin.

\section{Materials and Methods}

\section{Cell Culture}

Cultures of human primary epidermal keratinocytes derived from individual neonatal foreskins were initiated using the Rheinwald-Green system [Rheinwald and Green, 1975]. Human keratinocytes on mitomycin C-inactivated fibroblast feeder layers [Leigh and Watt, 1994] were expanded and used at the second or third passage for seeding on top of 2 day-old collagen-fibroblast matrices prepared as described earlier [Margulis et al., 2005]. Keratinocytes on these matrices were maintained under media until they were confluent, typically 3-4 days post-seeding. The kerati- 
nocyte/collagen-fibroblast unit was then raised to the air-liquid interface by placing it on top of stainless steel grids, the bottom surface of which was in direct contact with the media [Turksen et al., 1991]. Concurrent with this step, cultures were supplemented with vehicle as control (VEH; DMSO final concentration $0.1 \%$ ), CLF (1 mM; Sigma, St. Louis, Mo., USA), DHA (100 $\mu$ M; Sigma), WY (50 $\mu \mathrm{M}$; Chemsyn, Lenexa, Kans., USA), or retinoic acid (RA; $1 \mu \mathrm{M}$; Sigma) by addition to the media under the collagen-fibroblast matrix. Ligands were prepared as $1,000 \times$ final concentration stocks in DMSO. The media in control and ligand-treated cultures was renewed every second day. Similar PP responses detected by histological or biochemical methods (fig. 1-6) were obtained from organotypic cultures from each of three different donors.

PP concentrations were determined from initial titration experiments and literature reports for previous keratinocyte experiments or for selective activation of PPAR. We found no evidence of toxicity at $1 \mathrm{mM}$ CLF in contrast to that reported with its use at $500 \mu \mathrm{M}$ in low calcium (differentiation-limited), monolayer, submerged human keratinocyte cultures [Schmuth et al., 2004, and references therein] where their subsequent work was performed with $400 \mu \mathrm{M}$. A $1 \mathrm{mM}$ CLF treatment was previously used to ameliorate experimental contact dermatitis [Sheu et al., 2002] for twice daily applications in epidermal barrier function restoration studies and once daily applications for 5 of 6 days in mouse skin differentiation studies [Komuves et al., 2000]. DHA is considered a pan-PPAR agonist [Gani and Sylte, 2008, and references therein] although the effectiveness of receptor activation by DHA may be cell and species dependent [Seo et al., 2005]. Similar to CLF, WY has been used at a wide range of concentrations for keratinocyte studies, i.e. up to $1 \mathrm{~mm}$ for one time treatment following experimental irritation [Sheu et al., 2002], at $0.5 \mathrm{~mm}$ in twice daily applications for epidermal barrier function restoration studies, and at $0.5 \mathrm{mM}$ in once daily applications for 5 of 6 days in mouse skin differentiation studies [Komuves et al., 2000]. WY has been used at $100 \mu \mathrm{M}$, twice the concentration used here, to modulate barrier induction in rodent fetal skin explants [Schmuth et al., 2004, and references therein]. As described in previous caspase studies [Rendl et al., 2002], other investigators' work with skin equivalents [see Heise et al., 2006, for example], and activation of endogenous human keratinocyte RA receptor (RAR) [Jiang et al., 2008], RA treatment was performed at $1 \mu \mathrm{M}$.

Histology, Immunohistochemistry, Immunofluorescence, and Microscopy

Organotypic cultures were fixed in Bouin's fluid on day 8 , dehydrated, and paraffin embedded. Involucrin and filaggrin were detected by rabbit polyclonal $(1: 10)$ and mouse monoclonal (1:100) antibodies (Biomedical Technologies; Stoughton, Mass., USA), respectively, with immunogold enhancement (GE Healthcare; Piscataway, N.J., USA) as described [Aneskievich and Fuchs, 1992] and nuclear contrast from hematoxylin staining. Proliferating cell nuclear antigen (PCNA) was detected with antibody clone PC10 (Zymed; Invitrogen, Carlsbad, Calif., USA). Primary antibodies for serine 82 phosphorylated HSP 27 (phospho-HSP; Santa Cruz Biotechnology, Inc., Santa Cruz, Calif., USA), ATP-binding cassette, sub-family A (ABC1), member 12 (ABCA12), (Santa Cruz Biotechnology), and serine 473 phosphorylated Akt (phospho-Akt; Cell Signaling Technology, Danvers, Mass., USA) were detected with Alexa Fluor 488-tagged secondary antibody (Invi- trogen). Citrate buffer antigen retrieval was performed for PCNA, phospho-HSP, ABCA12, and phospho-Akt. Slides for fluorescence were mounted in ProLong Gold antifade reagent (Invitrogen). Digital images were captured using Spot 4.5.9.12 software and a Spot Insight color CCD or Spot Insight monochrome digital cameras. Exposure times and figure preparation were kept constant within detection of any one antigen.

\section{Electrophoresis and Western Detection}

Total proteins were extracted as described previously [Aneskievich and Fuchs, 1992] from the epidermal layer of organotypic cultures. Fifty micrograms of protein per lane were resolved through $8.5 \%$ denaturing polyacrylamide gels (12\% for caspase analysis) under reducing conditions, transferred to S\&S nitrocellulose (Fisher Scientific, Pittsburgh, Pa., USA), and Western probed with antibodies for filaggrin, keratin 13 (MP Biomedical, Aurora, Ohio, USA), RAR $\gamma$, retinoid X receptor (RXR) $\alpha$, caspase 3 , serine 82 phospho-HSP27 (all from Santa Cruz Biotechnology), total HSP27 (Enzo Life Sciences, Plymouth Meeting, Pa., USA), Akt, phospho-Akt (serine 473, both from Cell Signaling), or actin (Abcam, Cambridge, Mass., USA). Bound primary antibodies and SDS-PAGE MagicMark (Invitrogen) migration standards were visualized by HRP-conjugated secondary antibodies (PerkinElmer, Boston, Mass., USA).

Chemiluminescence was detected with an Image Station 440CF (Kodak, Rochester, N.Y., USA) CCD camera. Molecular weights and band intensities for relative comparisons of protein amounts were determined with Kodak MI analysis software directly from the original digital signal with actin used as a loading control.

\section{Northern Analysis}

PolyA-selected mRNA, $5 \mu \mathrm{g}$ per lane, was assayed with random primer probes generated from partial clones of human cDNAs for acyl-CoA oxidase (ACO; provided by J. Reddy), profilaggrin (provided by R. Presland), and GAPDH. Blots were washed under stringent conditions [Flores et al., 2005] and imaged by exposure to film at $-70^{\circ} \mathrm{C}$. GAPDH was used as a loading control.

\section{Statistical Analysis}

All experiments were repeated from different donors of keratinocytes with similar results. For statistical analysis, cultures were set in replicate samples and representative results are shown as means \pm standard error. Comparisons were performed using a one-way analysis of variance and Dunnet's test. Differences were considered statistically significant at $\mathrm{p}<0.05$.

\section{Results}

\section{Analysis of Organotypic Cultures: Morphometry,}

Structural Proteins, and Proliferation

Human keratinocytes atop fibroblast-collagen matrices were cultured at the air-media interface for 8 days, producing multiple distinct layers of keratinocytes (fig. 1a) with histological markers of late differentiation enucleated squames in the uppermost layers. There was increased stratification, especially in the granular and 

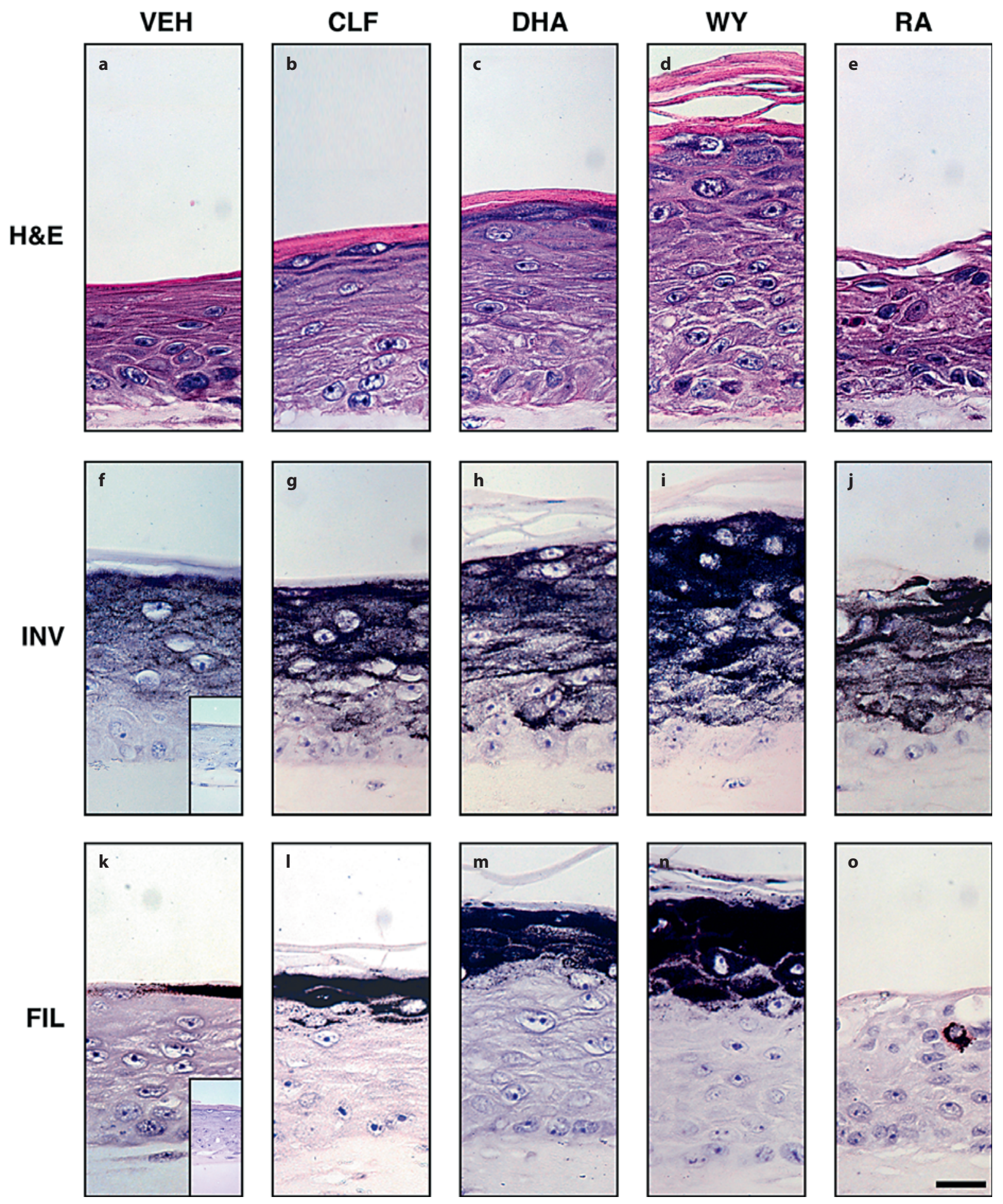

Fig. 1. Effects of PPs or RA on stratification and terminal differentiation of human keratinocytes in organotypic cultures. Cultures were fixed on day 8 and sections were processed for $\mathrm{H} \& \mathrm{E}$ staining (H\&E) (a-e), immunohistochemical detection of involucrin (INV) (f-j), or filaggrin (FIL) (k-o) with hematoxylin counterstaining. Treatment conditions were: vehicle control (VEH) (a,

f, k; DMSO final concentration 0.1\%); $1 \mathrm{mM} \mathrm{CLF} \mathrm{(b,} \mathrm{g,} \mathrm{I);} 100 \mu \mathrm{M}$ DHA (c, h, m); $50 \mu \mathrm{M} \mathrm{WY}(\mathbf{d}, \mathbf{i}, \mathbf{n})$, or $1 \mu \mathrm{M}$ RA $(\mathbf{e}, \mathbf{j}, \mathbf{o})$. Scale bar in $\mathbf{o}$ is $20 \mu \mathrm{m}$. Insets in $\mathbf{f}$ and $\mathbf{k}$ are negative controls for involucrin and filaggrin immunodetection, respectively; the scale bar for these is $60 \mu \mathrm{m}$. 

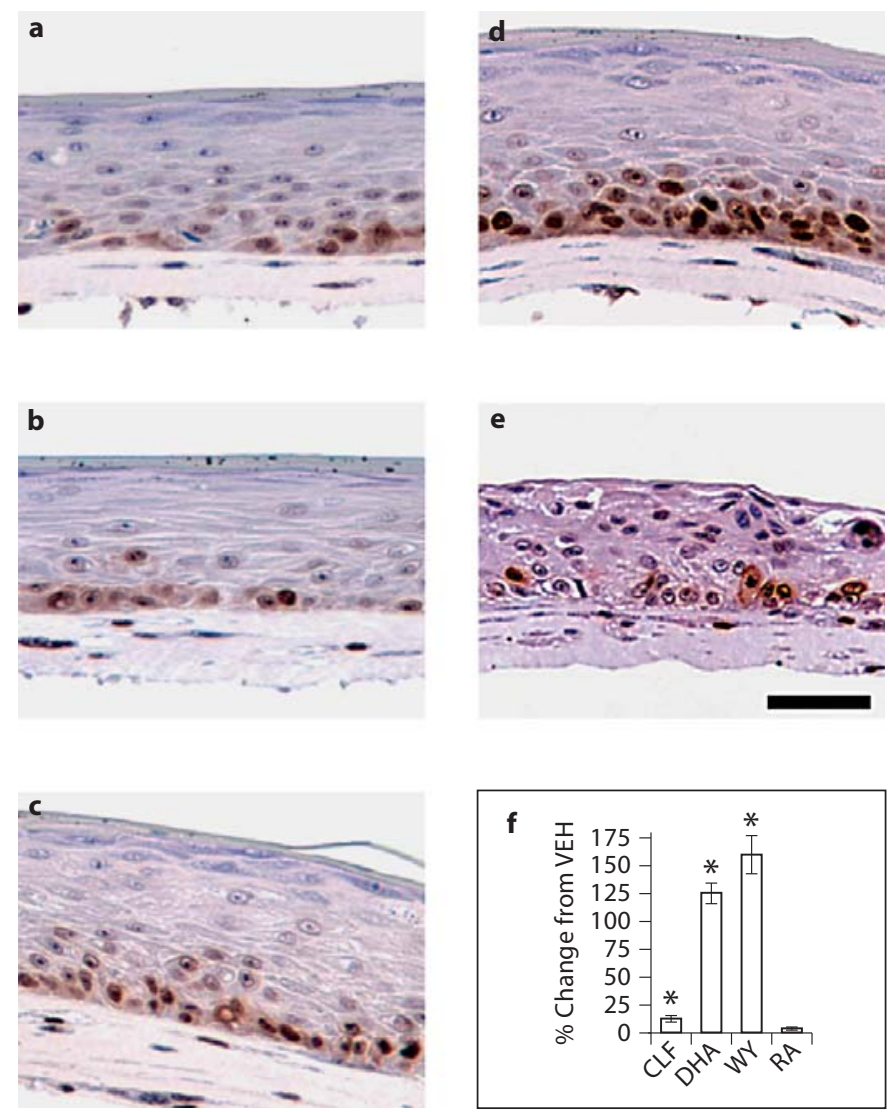

Fig. 2. Effects of PPs or RA on replication of human keratinocytes in organotypic cultures. Culture time and treatments as in figure 1. a Vehicle control (VEH). b CLF. c DHA. d WY. e RA. Immunohistochemical detection of PCNA was followed by hematoxylin counterstaining. Scale bar in e is $50 \mu \mathrm{m}$. Representative sections are shown. $\mathbf{f}$ The percent change of PCNA-positive cells compared to vehicle control cultures was determined from cells counted along three $200 \mu \mathrm{m}$ lengths from nonadjacent sections of the embedded culture. Results are from three cultures of independent keratinocyte donors and are presented as means \pm SEM; ${ }^{*} \mathrm{p} \leq$ 0.05 , Dunnett's test of ligands against vehicle.

cornified layers, for cultures where PPs had been added to the underlying media (fig. $1 \mathrm{~b}-\mathrm{d}$ ). Exposure to RA decreased the keratinization process (fig. 1e). In control cultures, involucrin staining was present in the first and subsequent suprabasal layers (fig. 1f). For PP- and RA-treated cultures, involucrin staining was more intense but remained restricted to the expected suprabasal layer compartment (fig. 1g-j). While filaggrin protein was restricted to the uppermost keratinocyte layer immediately exposed to the air in the control keratinocytes (fig. 1k), it was more intense and extended across more upper cell layers in the PP-treated cells (fig. 11-n), consistent with the more pronounced granular layer seen in the H\&E staining (fig. $1 \mathrm{~b}-\mathrm{d}$ ). Together, these results are consistent with PP treatment increasing overall keratinocyte differentiation (see Discussion) in contrast to RA treatment with reduced late maturation as evidenced by the rarely occurring filaggrin-positive cells (fig. 1o). These RA results are consistent with and extend monolayer keratinocyte expression studies [Poumay et al., 1999; Lee et al., 2009] that demonstrate that RA treatment can increase early maturation markers such as involucrin (fig. 1j) while repressing later markers of keratinocyte terminal differentiation such as filaggrin (fig. 10).

Compared to control cultures (fig. 2a), CLF-treated cultures, which had the least increase in epidermal thickening, showed the least PCNA staining increase (fig. 2b). DHA and WY induced striking hyperplastic effects, about doubling the overall epidermal thickness (fig. 1c, d) compared to the control culture (fig. 1a). For these cultures, PCNA staining (fig. 2c, d, respectively) was also significantly increased. The incidence of PCNA staining in the RA-treated cultures was not significantly changed from the control (fig. 2e, f).

\section{Distribution and Biochemical Analysis of \\ Differentiation-Related Proteins}

Development of a pronounced cornified layer and increased filaggrin staining indicated that PP treatment had enhanced structural aspects of keratinocyte late, terminal differentiation. We also tested for possible changes in staining intensity and distribution of nonstructural proteins associated with differentiation. The first was ABCA12; its expression is increased in submerged keratinocytes by some PPs [Jiang et al., 2008]. Immunoreactivity for $\mathrm{ABCA} 12$ was detected as a gradient throughout the strata of control organotypic keratinocytes with the least staining in the basal layer, increasing through to the granular layer (fig. 3a). This pattern parallels intact skin [Thomas et al., 2009]. Increased ABCA12 staining was most noticeable in the expanded granular layers of the PP-treated cultures (fig. 3b-d). Compared to control cultures, RA-treated keratinocytes had reduced staining for ABCA 12 and no obvious gradient distribution of the protein (fig. 3e).

HSP27 phosphorylation (serine 82) occurs as a stress response and parallels increased cornification [O'Shaughnessy et al., 2007; Robitaille et al., 2010]. For our organotypic cultures, phospho-HSP27 detection was present but discontinuous across late-granular keratinocytes. There was also the occasional mid-granular layer phospho-HSP27-positive cell (fig. 3f). 


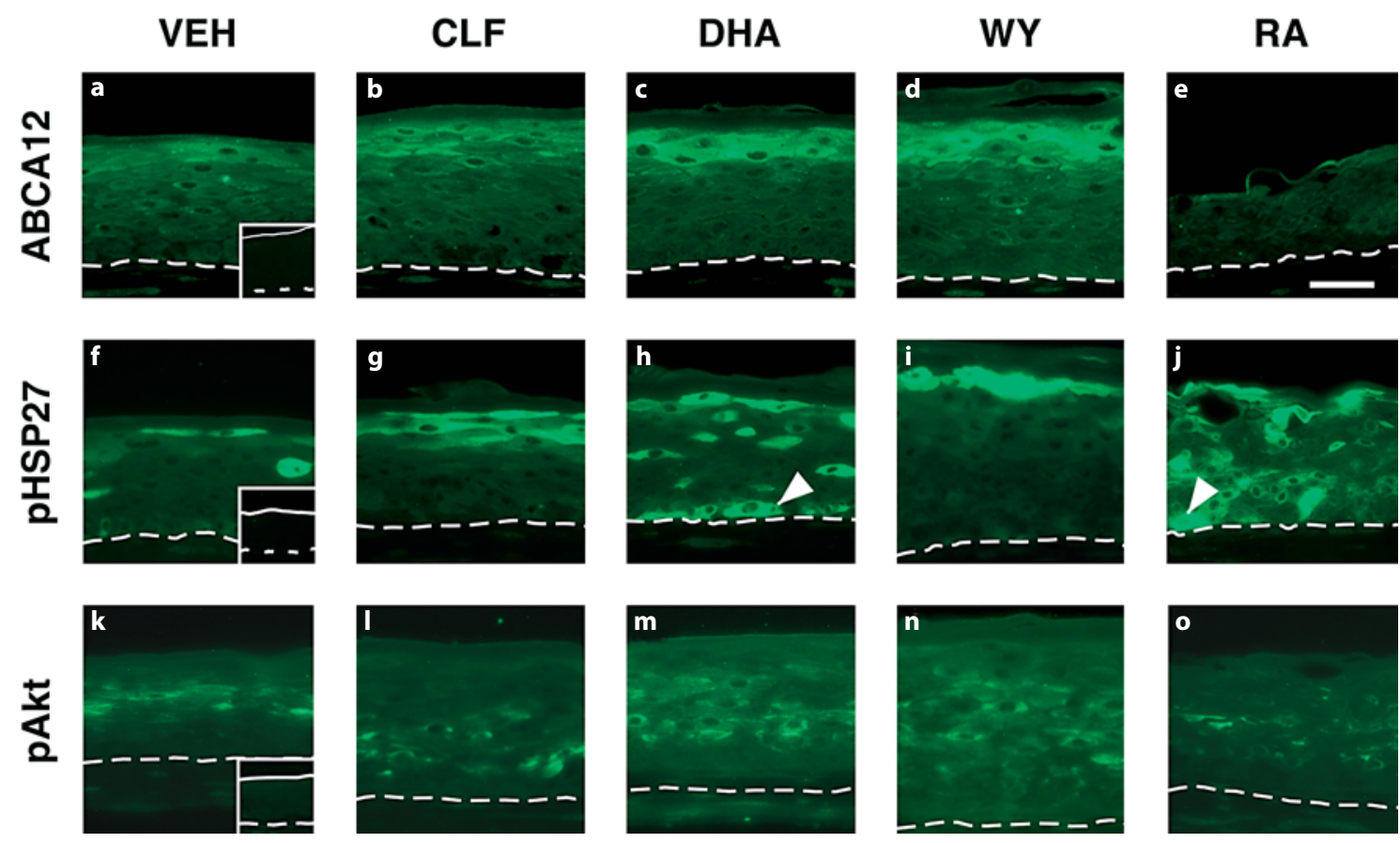

Fig. 3. Effects of PPs or RA on nonstructural proteins in human organotypic culture keratinocytes. Culture time and treatments as in figure 1. a-e ABCA12. f-j Phospho-HSP27 (pHSP27). k-o Phospho-Akt (pAkt). The dashed white line represents the keratinocyte-collagen boundary with the basal layer immediately above it. The scale bar in $\mathbf{e}$ is $50 \mu \mathrm{m}$. The insets in $\mathbf{a}, \mathbf{f}$, and $\mathbf{k}$ are negative controls for each primary antibody. Arrowheads in DHA (h) and RA (j) cultures point to intensely stained basal cells not present for pHSP in other treatments. Infrequent, intensely stained pHSP27 cells were noted for the vehicle condition as seen towards the right of panel $\mathbf{f}$. Use of other phospho-Akt antibodies or variant antigen retrieval protocols did not improve staining. Within each antigen set, including their respective negative controls shown in the insets, exposure times were kept identical. For the insets, the scale bar is $150 \mu \mathrm{m}$ and the dashed line is the keratinocyte-collagen boundary; the solid line is the surface of the epithelium.

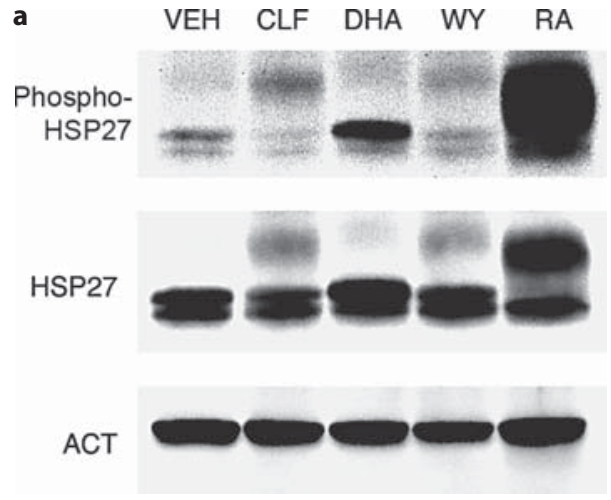

Fig. 4. Effects of PPs or RA on HSP27 and Akt protein levels. $\mathrm{VEH}=$ Vehicle control. Lysates for immunoblots were derived from sections of cultures shown in figure 3 and processed with antibodies specific for serine 82 phospho-HSP27 or total HSP27 (a) and serine 473 phospho-Akt or total Akt (b). Blots were re-

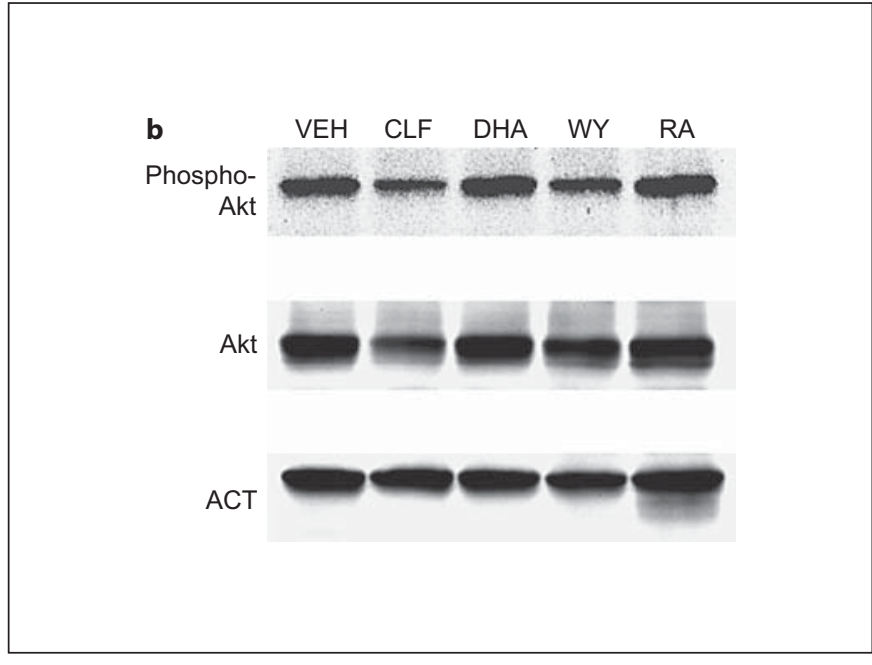

probed with anti-actin as a loading control. Anti-phospho-SER82 antibody recognizing the epitope in HSP27 phosphorylated at SER82 alone and with additional phosphorylated residues causing further reduction in migration may account for multiple band recognition by the antibody. 


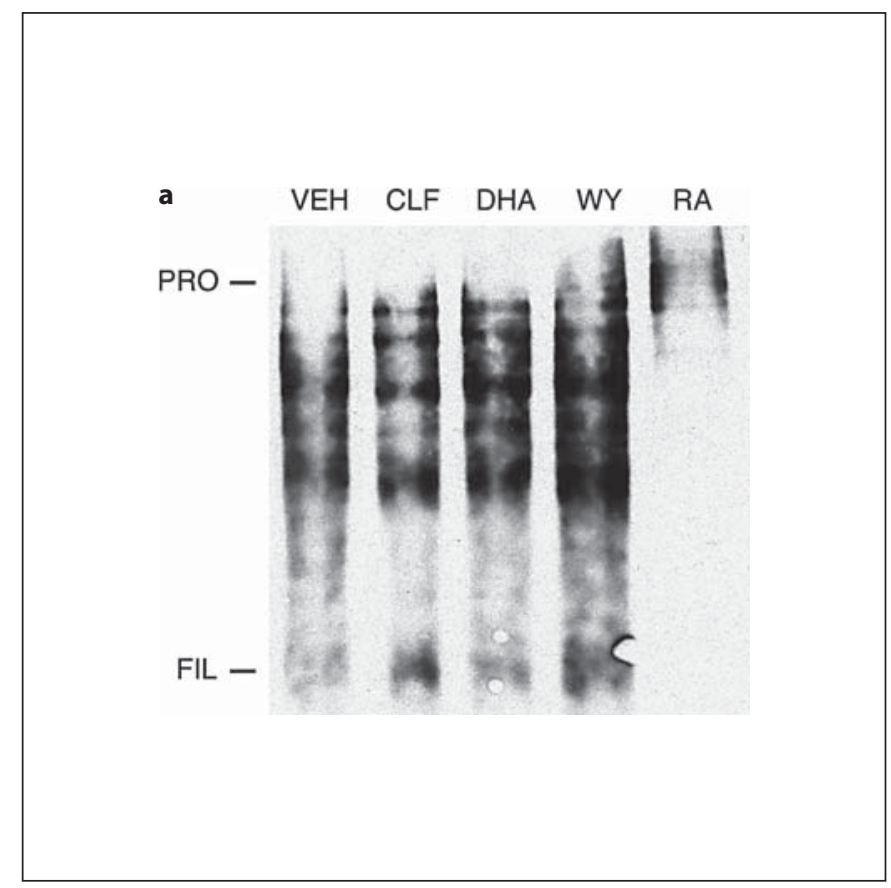

Fig. 5. Effects of PP or RA treatment on retinoid-regulated proteins. $\mathrm{VEH}=$ Vehicle control. Lysates for immunoblots were derived from sections of cultures shown in figure 3 and processed with antibodies specific for profilaggrin (PRO)/filaggrin (FIL) (a), keratin 13 (K13) (b), caspase 3 (CAS3) (c), RAR $\gamma(\mathbf{d}), \operatorname{RXR} \alpha(\mathbf{e})$,

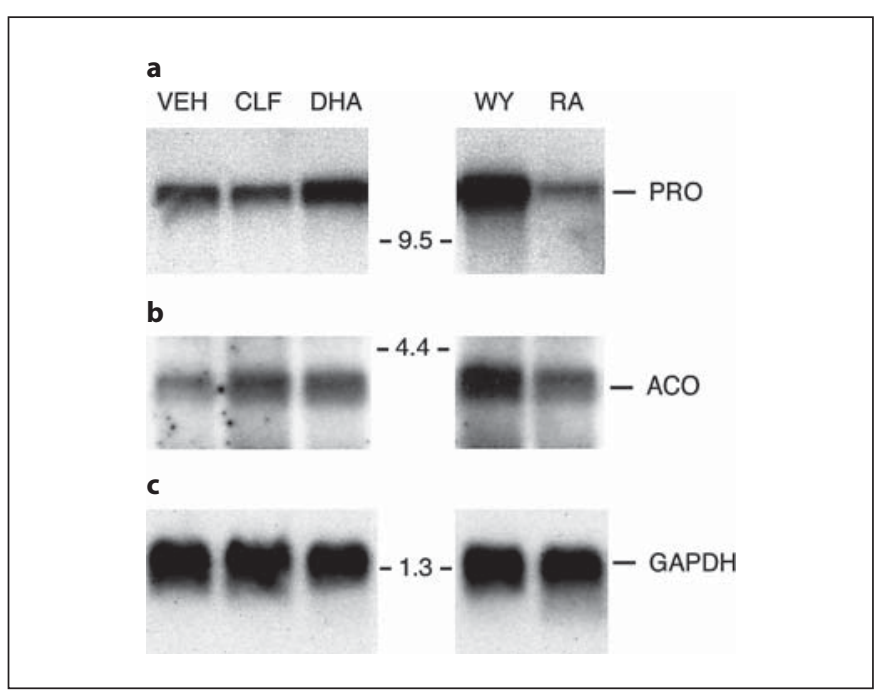

Fig. 6. PPs and RA regulation of keratinocyte mRNA levels. Northern analysis was conducted with poly A mRNA. Culture time and treatments as in figure 1. Blots were sequentially hybridized with probes based on human cDNAs for profilaggrin (PRO) (a), ACO (b), and glyceraldehyde-3-phosphate dehydrogenase (c), with GAPDH as a loading control. Samples were all run on the same gel where size markers separated vehicle (VEH), CLF-, and DHA-treated samples from WY and RA samples.

Keratinocyte Organotypic Modeling

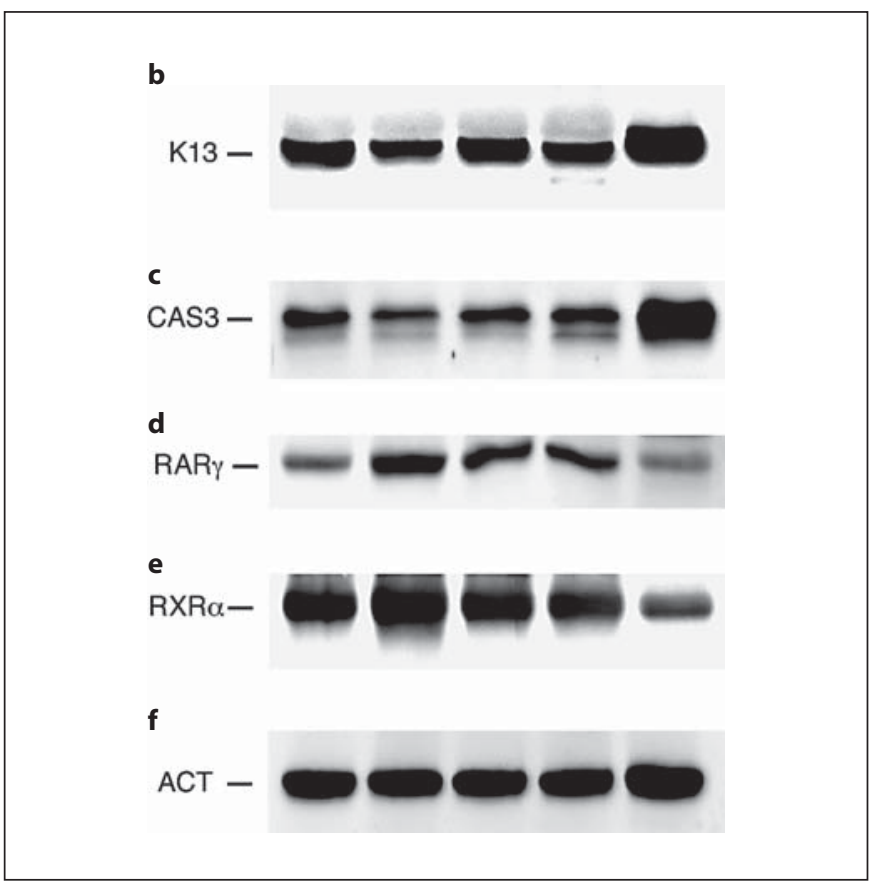

and actin $(\mathrm{ACT})(\mathbf{f})$. Band intensity changes were determined from digital imaging of the chemiluminescent signal using the actin loading control for normalization. Note that profilaggrin is a high molecular mass precursor sequentially enzymatically processed to the final smaller mass of filaggrin.

Compared to the vehicle control, phospho-HSP27 detection in CLF-, DHA-, and WY-treated cultures was more consistent across the granular layer (fig. $3 g-i$ ) with more frequently occurring intensely stained cells. Strikingly, several basal and spinous layer keratinocytes were strongly reactive for phospho-HSP27 (fig. 3h, arrowhead) in DHA-treated cultures, a result not seen for any other PP treatment. For WY cultures, the most intensely stained cells were routinely found in the first subcorneal layer. In the RA-treated cultures, moderately to intensely staining cells, much more numerous than in the control culture, were scattered through all layers, including frequent, intensely stained basal cells (fig. 3 j, arrowhead), suggesting an induction of phospho-HSP27 outside that associated with differentiation. These findings were mirrored in the biochemical analysis of phospho-HSP27 in the context of total HSP27 (fig. 4).

HSP phosphorylation is mediated in part by members of the protein kinase group. $\mathrm{PKB}$, also known as Akt, is activated when phosphorylated at serine 473 [Thrash et al., 2006] and in some cases [Di-Poi et al., 2002; Burdick et al., 2007] is present at increased levels following PP treatment. We found that basal keratinocytes in control, 
CLF, DHA, and RA cultures had weak or no staining (fig. 3k-o) for phospho-Akt (serine 473) compared to the secondary antibody only (fig. 3k, inset). WY-treated cultures (fig. 3n) routinely had weak staining throughout the basal layer. Otherwise, phospho-Akt distribution paralleled the PP-associated expansion of the spinous and granular layers with some increased intensity compared to the control. The total phospho-HSP27 signal increased 1.2- to 1.4-fold in the CLF-, DHA-, and WY-treated cultures; there was at least a 7-fold increase in the RA-treated samples (fig 4a). When normalized to the total Akt protein present in keratinocyte lysates from the organotypic cultures (fig. $4 \mathrm{~b}$ ), there was a 1.2- to 1.4-fold increase in phospho-Akt for PP-treated cultures relative to the vehicle control. No increase in phospho-Akt was found in lysates from the RA-treated cells.

Increases in filaggrin protein detected by immunoblot (fig. 5a) following PP treatment paralleled those seen with immunohistochemistry (fig. 1l-n), with the WY-treated culture showing the greatest amount $(\sim 3$-fold compared to the vehicle control). The increases were spread across both pro-filaggrin and the processed monomer form. RA treatment caused an almost complete loss of filaggrin. Not all keratinocyte-expressed proteins are decreased by RA and, in contrast to the filaggrin results, RA treatment induced an approximate 2 -fold K13 increase compared to vehicle control cultures, while the synthetic PPs CLF and WY each brought about decreases of approximately one half, with no change in the fatty acid DHA-treated culture (fig. 5b). RA also regulates differentiation-dependent markers other than structural proteins in epidermal keratinocytes. Exposure to RA resulted in $\sim 2.5$-fold more caspase 3 protein compared to control cultures, confirming previous reports of RA-mediated increases in keratinocyte organotypic cultures [Rendl et al., 2002]. In contrast, exposure to any of the three PPs caused a consistent one third to one half reduction of caspase 3 protein (fig. 5c). RAR $\gamma$ (NR1B3) and RXR $\alpha$ (NR2B1) are the predominant nuclear retinoid receptors expressed in epidermal keratinocytes. However, the PP-associated decreases in $\mathrm{K} 13$ and caspase 3 were not simply due to a loss of retinoid receptors (fig. 5d, e). For the PP-treated cultures, there was up to a 2-fold increase in RAR $\gamma$ protein $(\mathrm{CLF}>\mathrm{DHA} \approx \mathrm{WY})$ compared to control cultures. $\mathrm{RXR} \alpha$, the heterodimer to both RARs and PPARs, showed some variation with an $\sim 20 \%$ increase in CLF cultures and an $\sim 10 \%$ decrease in WY-treated keratinocytes. Consistent with previous reports [Boudjelal et al., 2002], RA exposure reduced both RAR $\gamma$ and RXR $\alpha$ protein levels.
Expression changes due to PP or RA exposure were also examined at the mRNA level. In agreement with the increase in filaggrin protein, there was a PP-dependent increase (fig. $6 \mathrm{a}$ ) in an $\sim 13-\mathrm{kb}$ profilaggrin mRNA band. As with the changes in filaggrin protein levels, DHA and WY had the greatest increases in profilaggrin mRNA levels with a reduction in signal from the RA-treated cultures compared to vehicle controls. The ACO promoter has a recognized PP response element and its expression (fig. 6b) was increased by the three PPs compared to control cultures. RA treatment induced a lesser ACO increase.

\section{Discussion}

The studies described here utilized organotypic cultures and their ability to recapitulate structural and biochemical markers of epidermal differentiation with high fidelity [Margulis et al., 2005] to investigate human keratinocyte responses to PPAR $\alpha$ agonists and RA. CLF, WY, and DHA increased stratification and cornification. PPs and RA oppositely affected filaggrin and K13. PP-dependent changes in retinoid-sensitive markers (increase in retinoid-repressed filaggrin; decrease in retinoid-enhanced markers K13) could not be simply attributed to loss of retinoid receptor protein, suggesting that a balance of these ligands is needed for differentiation.

Although evidenced to different degrees, the three PPs used shared the same trend of moderate to significantly increased keratinocyte replication and enhanced differentiation. For instance, the synthetic PPAR $\alpha$ agonists CLF and WY produced similar but different degrees of effects for the late markers filaggrin and ABCA12; both ligands increased both proteins but with the most enhanced effect from WY treatment. This is consistent with and may hold true across species for previous comparisons of the two compounds where, although CLF was used at a 10-fold higher final concentration than WY, the effects of WY were of a greater magnitude [Motojima, 2000], such as with the higher fold induction of heart fatty acid binding protein. Effects of DHA are likely to extend beyond its pan-PPAR agonist function. For instance, HaCaT keratinocytes grown in DHA-supplemented media showed physical incorporation of this fatty acid into their cellular constituents [Storey et al., 2005] possibly altering their cell membrane properties and other signaling events.

The keratinocyte growth-promoting effect from ligand activation of PPAR $\alpha$ in this report is distinct from 
that described for rodent epidermis where the bovine keratin 5 promoter was used to direct basal cell expression of a constitutively activated, viral protein-mouse PPAR $\alpha$ chimera (VP16PPAR $\alpha$ ) which resulted in a thinning of the epidermal layer [Yang et al., 2006]. Enhanced differentiation but decreased proliferation in the VP16PPAR $\alpha$ transgenic mouse system may reflect species differences [Lawrence et al., 2001; Kane et al., 2006; Al Kholaifi et al., 2008] and/or the cohort of transcriptional coactivators recruited by PPAR in an activated conformation due to ligand binding versus transcriptional activation as a result of the viral protein activation domain. The PP-enhanced growth in organotypic cultures also differed from the CLF-reduced growth for keratinocytes grown directly on plastic, submerged in low calcium media [Hanley et al., 1998; Schmuth et al., 2004]. Nevertheless, involucrin detection, indicative of cell cycle withdrawal and terminal differentiation onset in the organotypic cultures, still began at the appropriate, first suprabasal layer. This pattern suggests that PP growth promotion did not abrogate the usual stratum-specific events associated with progressive keratinocyte maturation. Thus the keratinocyte culture context (submerged vs. organotypic) or the means of receptor activation (ligand vs. viral protein activation) may influence the cell's PP/PPAR response. Understandably, each experimental system, keratinocyte monoculture, organotypic culture, or wild-type or genetically modified mouse, will have its advantages and limitations depending on what aspects of keratinocyte biology are being investigated.

Late in epidermal maturation, ABCA12 participates in transport of lipids into lamellar bodies. As with the differentiation marker filaggrin, we found increases in ABCA12 in histological sections of the organotypic cultures treated with CLF, DHA, or WY. Keratinocyte ABCA12 mRNA has been reported to increase [Jiang et al., 2008] following exposure to agonists for PPAR $\delta$ and $\gamma$ but not WY. Several experimental differences exist between that report [Jiang et al., 2008] and this study that may account for the different WY results including duration of ligand treatment ( $24 \mathrm{~h}$ vs. multiple days) and the nature of the keratinocyte culture environment (submerged, providing basal and early differentiation stages vs. organotypic, recapitulating all epidermal strata).

As expected from the increased degree of differentiation demonstrated in the histology and structural protein biochemistry, we found that CLF, DHA, and WY treatment also increased phospho-HSP27 levels. However, with DHA or RA treatment we frequently detected intensely staining basal cells, and within the context of total
HSP27 protein there was a several-fold increase in phospho-HSP27 following RA exposure. Some HSP27 phosphorylation may be differentiation independent. Phospho-HSP27 (serine 82) increases in MDA-MB-435 cells and epidermal squamous cell carcinoma keratinocytes following their exposure to arachidonic acid or the antineoplastic synthetic retinoid fenretinide, respectively, [Kim et al., 2006; Garcia et al., 2009]. Thus some increased phospho-HSP27 may be an indication of cells responding to stimuli independently of whether those signals influence differentiation [Kostenko and Moens, 2009].

Akt is one among multiple kinases responsible for HSP27 phosphorylation [Kostenko and Moens, 2009]. Phospho-Akt coincides with late differentiation in mouse epidermis [O'Shaughnessy et al., 2007] and its signaling can be increased in mouse monolayer keratinocyte cultures treated with a PPAR $\delta$ ligand [Di-Poi et al., 2002]. However, treatment of an hTERT-immortalized human keratinocyte cell line in submerged cultures with the PPAR $\delta$ ligand GW0742 led to no phospho-Akt or total Akt differences [Burdick et al., 2007], suggesting possible species-specific effects. We found a consistent but less than 2-fold increase in levels of phospho-Akt protein and phospho-HSP27. However, staining intensity for phospho-Akt (early spinous and early granular) was localized to mostly different layers compared to phsopho-HSP27 (late-granular for CLF and WY), suggesting that some other kinases, such as mitogen-activated protein kinase (MAPK)-activated protein kinase 2 [Kostenko and Moens, 2009], may be involved in increased phosphoHSP27 levels.

Dubrac and Schmuth [Dubrac and Schmuth, 2006] noted that while rodent studies may be illustrative of some human cutaneous responses to PPs there are likely limits due to species specificity. Even among rodents, there is variation in response to PPs [see Choudhury et al., 2000, for example; Al Kholaifi et al., 2008]. Several experimental factors discussed here and by others [Burdick et al., 2006; Peraza et al., 2006] such as variations in assay systems, species, ligand, and PPAR isoform may restrict the prediction of one universally expected biological response (e.g. growth vs. differentiation or upregulation of a specific marker) in all cell types across diverse tissues.

The wide range of ligands for and wide expression distribution of PPAR have suggested their potential importance in a number of extra-hepatic processes including epidermal keratinocyte growth and differentiation which in turn would impact normal tissue turnover, wound 
healing, and hyperplastic diseases. Animal studies with clinical and laboratory PPAR $\alpha$ ligands, such as CLF and WY, in wild-type and recombinant mouse strains, complemented by standard human keratinocyte cultures provide experimentally distinct venues for PP investigation, each with their particular advantages and limitations. In this context, our results suggest there may be species-specific keratinocyte responses as well as limitations of standard culture methods that can be addressed though organotypic models of human keratinocytes.

\section{Acknowledgements}

This work received grant support from the American Institute for Cancer Research, NIAMS (AR048660), and the Connecticut Stem Cell Research Program (Connecticut Innovations). We thank the journal reviewers and editor for helpful suggestions in improving this report.

\section{References}

Al Kholaifi A., A. Amer, B. Jeffery, T.J. Gray, R.A. Choudhury A.I., Chahal S., Bell A.R., Tomlinson Roberts, D.R. Bell (2008) Species specific kinetics and zonation of hepatic DNA synthesis induced by ligands of PPARalpha. Toxicol Sci 104: 74-85.

-Aneskievich B.J., E. Fuchs (1992) Terminal differentiation in keratinocytes involves positive as well as negative regulation by retinoic acid receptors and retinoid $\mathrm{X}$ receptors at retinoid response elements. Mol Cell Bio 12: 4862-4871.

Batheja P., Y. Song, P. Wertz, B. Michniak-Kohn (2009) Effects of growth conditions on the barrier properties of a human skin equivalent. Pharm Res 26: 1689-1700.

Boudjelal M., J.J. Voorhees, G.J. Fisher (2002) Retinoid signaling is attenuated by proteasome-mediated degradation of retinoid receptors in human keratinocyte HaCaTcells. Exp Cell Res 274: 130-137.

Burdick A.D., M.T. Bility, E.E. Girroir, A.N. Billin, T.M. Willson, F.J. Gonzalez, J.M. Peters (2007) Ligand activation of peroxisome proliferator-activated receptor-beta/ delta(PPARbeta/delta) inhibits cell growth of human N/TERT-1 keratinocytes. Cell Signal 19: 1163-1171.

Burdick A.D., D.J. Kim, M.A. Peraza, F.J. Gonzalez, J.M. Peters (2006) The role of peroxisome proliferator-activated receptor-beta/delta in epithelial cell growth and differentiation. Cell Signal 18: 9-20.

Cheung C., T.E. Akiyama, J.M. Ward, C.J. Nicol, L. Feigenbaum, C. Vinson, F.J. Gonzalez (2004) Diminished hepatocellular proliferation in mice humanized for the nuclear receptor peroxisome proliferator-activated receptor alpha. Cancer Res 64: 3849-3854.

Chong H.C., M.J. Tan, V. Philippe, S.H. Tan, C.K Tan, C.W. Ku, Y.Y. Goh, W. Wahli, L. Michalik, N.S. Tan (2009) Regulation of epithelialmesenchymal IL-1 signaling by PPARbeta/ delta is essential for skin homeostasis and wound healing. J Cell Biol 184: 817-831. S.R., Roberts R.A., Salter A.M., Bell D.R. (2000) Species differences in peroxisome proliferation: mechanisms and relevance. Mutat Re 448: 201-222.

Di-Poi N., N.S. Tan, L. Michalik, W. Wahli, B. Desvergne (2002) Antiapoptotic role of PPARbeta in keratinocytes via transcriptional control of the Akt1 signaling pathway. Mol Cell 10: 721-733.

Dubrac S., M. Schmuth (2006) (P)PARsing epidermal development. J Invest Dermatol 126: 241-242.

Flores A.M., L. Li, N.G. McHugh, B.J. Aneskievich (2005) Enzyme association with PPARgamma: evidence of a new role for 15-lipoxygenase type 2. Chem Biol Interact 151: $121-$ 132.

Gani O.A., I. Sylte (2008) Molecular recognition of docosahexaenoic acid by peroxisome proliferator-activated receptors and retinoid-X receptor alpha. J Mol Graph Model 27: 217224.

Garcia M.C., D.M. Ray, B. Lackford, M. Rubino, K. Olden, J.D. Roberts (2009) Arachidonic acid stimulates cell adhesion through a novel p38 MAPK-RhoA signaling pathway that involves heat shock protein 27. J Biol Chem 284: 20936-20945.

Gonzalez F.J., Y.M. Shah (2008) PPARalpha: mechanism of species differences and hepatocarcinogenesis of peroxisome proliferators. Toxicology 246: 2-8.

Hanley K., Y. Jiang, S.S. He, M. Friedman, P.M. Elias, D.D. Bikle, M.L. Williams, K.R. Feingold (1998) Keratinocyte differentiation is stimulated by activators of the nuclear hormone receptor PPARalpha. J Invest Dermatol 110: 368-375.

Heise R., J. Mey, M.M. Neis, Y. Marquardt, S. Joussen, H. Ott, T. Wiederholt, P. Kurschat, M. Megahed, D.R. Bickers, H.F. Merk, J.M. Baron (2006) Skin retinoid concentrations are modulated by CYP26AI expression restricted to basal keratinocytes in normal human skin and differentiated 3D skin models. J Invest Dermatol 126: 2473-2480.
Jiang Y.J., B. Lu, P. Kim, G. Paragh, G. Schmitz, P.M. Elias, K.R. Feingold (2008) PPAR and LXR activators regulate ABCA12 expression in human keratinocytes. J Invest Dermatol 128: 104-109.

Kane C.D., O.L. Francone, K.A. Stevens (2006) Differential regulation of the cynomolgus, human, and rat acyl-CoA oxidase promoters by PPARalpha. Gene 380: 84-94

Keller H., P.R. Devchand, M. Perroud, W. Wahli (1997) PPAR alpha structure-function relationships derived from species-specific differences in responsiveness to hypolipidemic agents. Biol Chem 378: 651-655.

Kim H.J., N. Chakravarti, N. Oridate, C. Choe, F.X. Claret, R. Lotan (2006) N-(4-hydroxyphenyl)retinamide-induced apoptosis triggered by reactive oxygen species is mediated by activation of MAPKs in head and neck squamous carcinoma cells. Oncogene 25: 2785-2794.

Klaunig J.E., M.A. Babich, K.P. Baetcke, J.C. Cook, J.C. Corton, R.M. David, J.G. DeLuca, D.Y. Lai, R.H. McKee, J.M. Peters, R.A. Roberts, P.A. Fenner-Crisp (2003) PPARalpha agonist-induced rodent tumors: modes of action and human relevance. Crit Rev Toxicol 33: $655-780$

Komuves L.G., K. Hanley, A.M. Lefebvre, M.Q. Man, D.C. Ng, D.D. Bikle, M.L. Williams, P.M. Elias, J. Auwerx, K.R. Feingold (2000) Stimulation of PPARalpha promotes epidermal keratinocyte differentiation in vivo. J Invest Dermatol 115: 353-360.

Kostenko S., U. Moens (2009) Heat shock protein 27 phosphorylation: kinases, phosphatases, functions and pathology. Cell Mol Life Sci 66: 3289-3307.

Lawrence J.W., Y. Li, S. Chen, J.G. DeLuca, J.P. Berger, D.R. Umbenhauer, D.E. Moller, G. Zhou (2001) Differential gene regulation in human versus rodent hepatocytes by peroxisome proliferator-activated receptor (PPAR) alpha. J Biol Chem 276: 31521-31527. 
Lee D.D., O. Stojadinovic, A. Krzyzanowska, C. Poumay Y., F. Herphelin, P. Smits, I.Y. De Potter, Vouthounis, M. Blumenberg, M. Tomic-Canic (2009) Retinoid-responsive transcriptional changes in epidermal keratinocytes. J Cell Physiol 220: 427-439.

Leigh I.M., F.M. Watt (1994) Keratinocyte Methods. Cambridge, Cambridge University Press.

Maloney E.K., D.J. Waxman (1999) trans-Activation of PPARalpha and PPARgamma by structurally diverse environmental chemicals. Toxicol Appl Pharmacol 161: 209-218.

Margulis A., W. Zhang, J.A. Garlick (2005) In vitro fabrication of engineered human skin. Methods Mol Biol 289: 61-70.

Michalik L., J. Auwerx, J.P. Berger, V.K. Chatterjee, C.K. Glass, F.J. Gonzalez, P.A. Grimaldi, T. Kadowaki, M.A. Lazar, S. O’Rahilly, C.N. Palmer, J. Plutzky, J.K. Reddy, B.M. Spiegelman, B. Staels, W. Wahli (2006) International Union of Pharmacology. 61. Peroxisome proliferator-activated receptors. Pharmacol Rev 58: 726-741.

Michalik L., W. Wahli (2007) Peroxisome proliferator-activated receptors (PPARs) in skin health, repair and disease. Biochim Biophys Acta 1771: 991-998.

Morimura K., C. Cheung, J.M. Ward, J.K. Reddy, F.J. Gonzalez (2006) Differential susceptibility of mice humanized for peroxisome proliferator-activated receptor alpha to $\mathrm{Wy}$ 14,643-induced liver tumorigenesis. Carcinogenesis 27: 1074-1080.

Motojima K. (2000) Differential effects of PPARalpha activators on induction of ectopic expression of tissue-specific fatty acid binding protein genes in the mouse liver. Int J Biochem Cell Biol 32: 1085-1092.

O’Shaughnessy R.F., J.C. Welti, J.C. Cooke, A.A. Avilion, B. Monks, M.J. Birnbaum, C. Byrne (2007) AKT-dependent HspB1 (Hsp27) activity in epidermal differentiation. J Biol Chem 282: 17297-17305.

Peraza M.A., A.D. Burdick, H.E. Marin, F.J. Gonzalez, J.M. Peters (2006) The toxicology of ligands for peroxisome proliferator-activated receptors (PPAR). Toxicol Sci 90: 269295.

Peters J.M., C. Cheung, F.J. Gonzalez (2005) Peroxisome proliferator-activated receptor-alpha and liver cancer: where do we stand? J Mol Med 83: 774-785. M.R. Pittelkow (1999) High-cell-density phorbol ester and retinoic acid upregulate involucrin and downregulate suprabasal keratin 10 in autocrine cultures of human epidermal keratinocytes. Mol Cell Biol Res Commun 2: 138-144.

Rendl M., J. Ban, P. Mrass, C. Mayer, B. Lengauer, L. Eckhart, W. Declerq, E. Tschachler (2002) Caspase-14 expression by epidermal keratinocytes is regulated by retinoids in a differentiation-associated manner. J Invest Dermatol 119: 1150-1155.

Rheinwald J.G., H. Green (1975) Serial cultivation of strains of human epidermal keratinocytes: the formation of keratinizing colonies from single cells. Cell 6: 331-344.

Rivier M., I. Castiel, I. Safonova, G. Ailhaud, S. Michel (2000) Peroxisome proliferator activated receptor-alpha enhances lipid metabolism in a skin equivalent model. J Invest Dermatol 114: 681-687.

Robitaille H., C. Simard-Bisson, D. Larouche, R.M. Tanguay, R. Blouin, L. Germain (2010) The small heat-shock protein Hsp27 undergoes ERK-dependent phosphorylation and redistribution to the cytoskeleton in response to dual leucine zipper-bearing kinase expression. J Invest Dermatol 130: 74-85.

Schmuth M., C.M. Haqq, W.J. Cairns, J.C. Holder, S. Dorsam, S. Chang, P. Lau, A.J. Fowler, G. Chuang, A.H. Moser, B.E. Brown, M. Mao-Qiang, Y. Uchida, K. Schoonjans, J. Auwerx, P. Chambon, T.M. Willson, P.M. Elias K.R. Feingold (2004) Peroxisome proliferator-activated receptor (PPAR)-beta/delta stimulates differentiation and lipid accumulation in keratinocytes. J Invest Dermatol

Schmuth M., Y.J. Jiang, S. Dubrac, P.M. Elias, K.R. Feingold (2008) Thematic review series: skin lipids - peroxisome proliferator-activated receptors and liver $\mathrm{X}$ receptors in epidermal biology. J Lipid Res 49: 499-509.

Seo T., W.S. Blaner, R.J. Deckelbaum R.J. (2005) Omega-3 fatty acids: molecular approaches to optimal biological outcomes. Curr Opin Lipidol 16: 11-18.

Sheu M.Y., A.J. Fowler, J. Kao, M. Schmuth, K. Schoonjans, J. Auwerx, J.W. Fluhr, M.Q. Man, P.M. Elias, K.R. Feingold (2002) Topical peroxisome proliferator activated receptor alpha activators reduce inflammation in irritant and allergic contact dermatitis models. J Invest Dermatol 118: 94-101. 122: 971-983.
Storey A., F. McArdle, P.S. Friedmann, M.J. Jackson, L.E. Rhodes (2005) Eicosapentaenoic acid and docosahexaenoic acid reduce UVBand TNF-alpha-induced IL- 8 secretion in keratinocytes and UVB-induced IL-8 in fibroblasts. J Invest Dermatol 124: 248-255.

Takeuchi S., T. Matsuda, S. Kobayashi, T. Takahashi, H. Kojima (2006) In vitro screening of 200 pesticides for agonistic activity via mouse peroxisome proliferator-activated receptor (PPAR)alpha and PPARgamma and quantitative analysis of in vivo induction pathway. Toxicol Appl Pharmacol 217: 235244.

Thomas A.C., D. Tattersall, E.E. Norgett, E.A. O’Toole, D.P. Kelsell (2009) Premature terminal differentiation and a reduction in specific proteases associated with loss ofABCA12 in Harlequin ichthyosis. Am J Pathol 174: 970-978.

Thrash B.R., C.W. Menges, R.H. Pierce, D.J. McCance (2006) AKT1 provides an essential survival signal required for differentiation and stratification of primary human keratinocytes. J Biol Chem 281: 12155-12162.

Turksen K., Y. Choi, E. Fuchs (1991) Transforming growth factor alpha induced collagen degradation and cell migration in differentiating human epidermal raft cultures. Cell Regul 2: 613-625.

Varga T., Z. Czimmerer, L. Nagy (2011) PPARs are a unique set of fatty acid regulated transcription factors controlling both lipid metabolism and inflammation. Biochim Biophys Acta 1812: 1007-1022.

-Westergaard M., J. Henningsen, M.L. Svendsen, C. Johansen, U.B. Jensen, H.D. Schroder, I. Kratchmarova, R.K. Berge, L. Iversen, L. Bolund, K. Kragballe, K. Kristiansen (2001) Modulation of keratinocyte gene expression and differentiation by PPAR-selective ligands and tetradecylthioacetic acid. J Invest Dermatol 116: 702-712.

Yang Q., A. Yamada, S. Kimura, J.M. Peters, F.J. Gonzalez (2006) Alterations in skin and stratified epithelia by constitutively activated PPARalpha. J Invest Dermatol 126: 374385 